\title{
Design of a New Non-rare-earth Magnetic Variable Gear for Hybrid Vehicular Propulsion System
}

\author{
Mu Chen, K. T. Chau, Fellow, IET, and Chunhua Liu \\ Department of Electrical and Electronic Engineering, The University of Hong Kong, Hong Kong, China
}

This paper presents the design of a new non-rare-earth magnetic variable gear (MVG) with sixteen electrically controlled gear ratios, which incorporates both the concept of the magnetic gear and the concept of the memory machine. The high-remanence low-coercivity permanent magnet material, namely the aluminum-nickel-cobalt, is adopted to realize the controllable gear ratios. The key is to design the stationary ring newly integrated with magnetizing windings in such a way that sixteen sets of gear ratios can be achieved so as to fulfill different driving requirements and road conditions. Firstly, the original MVG, the improved MVG and the proposed MVG are discussed. Then, a comprehensive analysis of the proposed MVG is conducted. Consequently, the proposed MVG is extended to electronic-continuously variable transmission for hybrid vehicular propulsion. By using finite element analysis, the electromagnetic performances of three MVGs at various gear ratios are evaluated, hence validating the theoretical design. Moreover, the process of gear-ratio-changing is simulated and analyzed. A quantitative comparison among these MVGs is also carried out. Hence, the corresponding validity can be further verified.

Index Terms - Non-rare-earth, magnetic gear, gear-ratio-changing, electric vehicle, hybrid propulsion.

\section{INTRODUCTION}

DUE to great concerns on environmental pollution and energy crisis, hybrid electric vehicles (HEVs) have been identified to the most promising and practical solution for road transportation, and have attracted growing global interests in recent years [1][3]. In general, the HEVs are equipped with mechanical gears and gearboxes to coordinate the driving torques produced by the engine and the electric motor as well as to amplify the driveline torque or speed for various driving requirements and road conditions [4]-[6]. However, these mechanical gears and gearboxes inevitably suffer from the disadvantages of transmission loss, annoying noise and need of regular maintenance.

Recently, magnetic gears have been considered as the most promising candidate to compete with the traditional mechanical gears because of their merits of contactless operation, noise free and maintenance free [7]-[9]. Nevertheless, a direct replacement of mechanical gears by magnetic gears cannot solve the problems aroused by the mechanical gearboxes. In particular, normal 
magnetic gears can only offer a fixed gear ratio whereas the gear ratio of conventional vehicles needs to be flexibly changed to fulfill various driving requirements and road conditions.

There are considerable concerns on the supply and price of rare-earth permanent magnet (PM) materials such as samariumcobalt $(\mathrm{SmCo})$ and neodymium-iron-boron $(\mathrm{NdFeB})$, which are widely used in the existing magnetic gears. In order to assess the usefulness of non-rare-earth PMs for magnetic gears, a quantitative cost-effectiveness comparison between rare-earth PM and non-rare-earth PM based magnetic gears was conducted [10]. The results indicated that the non-rare-earth PM material aluminum-nickel-cobalt (Alnico) can offer the lowest price per unit torque developed and can provide sufficient torque density for industrial application. Furthermore, it pointed out that the major disadvantage of Alnico, namely the low coercivity, is not valid in normal magnetic gears because there is no armature field involved.

In addition, the key characteristic of the memory machine, namely the electrically controllable magnetization level, arouses great interest from researchers. The first concept of the memory machine originates from the wide-speed-range PM machine proposed by Ostovic [11]. The key of this AC-excited memory machine is that it purposely utilizes the d-axis component of the armature current to magnetize or demagnetize the Alnico PMs to different levels of magnetization, hence obtaining controllable air-gap flux density. Furthermore, the DC-excited memory machine is also proposed by inserting a small DC magnetizing winding into the stator-PM machine to perform the online magnetization or demagnetization of the Alnico PMs [12]-[13]. Generally, the DC-excited memory machine offers the merits of higher mechanical reliability and better controllability than the AC-excited counterpart.

This paper aims to combine the concept of magnetic gears and the concept of DC-excited memory machines to form a new non-rare-earth magnetic variable gear (MVG) for the hybrid vehicular propulsion system. The key is to artfully insert the magnetizing windings into the stationary ring in such a way the proposed MVG can offer a wide range of changeable gear ratios, namely sixteen gear ratios. By using finite element analysis, the electromagnetic performances of all three MVGs at different gear ratios will be analyzed and evaluated. Also, the process of gear-ratio-changing will be presented. Hence, the corresponding validity can be verified.

The key contribution of this paper is to propose an Alnico based MVG, having sixteen controllable gear ratios for the hybrid vehicular propulsion system, which incorporates the concept of magnetic gears and the concept of DC-excited memory machines. Compared with previous MVGs, the proposed MVG can solve the drawbacks of high torque ripple, high magnetizing current and complicated structure of the original MVG [14] as well as the drawbacks of high magnetizing current and very complicated structure of the improved MVG [15]. With the sixteen flexible gear ratios, the proposed MVG can be utilized to supersede the mechanical gearbox for hybrid vehicular propulsion. 


\section{Design of Magnetic VARIABle Gears}

Fig. 1(a) shows the configuration of the original MVG which consists of the outer rotor with surface mounted Alnico PM pieces, the stationary ring with magnetizing windings and the inner rotor with surface mounted Alnico PM pieces [14]. As shown in Fig. 1(a), the inner rotor adopts a smaller number of PM pole-pairs (4 pole-pairs), whereas the outer rotor adopts a larger number of PM pole-pairs (7 pole-pairs). The magnetizing windings are inserted into the stationary ring in such a way the gear ratios can be flexibly controlled. The key design parameters of this MVG are listed in Table I. This original MVG can offer six different gear ratios, namely from 0.38 to 2.67 , to meet different road conditions and driving requirements. Also the non-rareearth PM material Alnico, which takes the advantage of high Curie temperature, works well for harsh vehicular environment. However, as shown in Fig 1(a), the stationary ring is divided into small strips due to the introduction of magnetizing windings. It imposes negative influence on the field modulation effect. Hence this MVG exhibits the demerit of high torque ripple. Also, the corresponding magnetizing windings in the air part of the stationary ring needs high magnetizing current to tune the magnetization level of Alnico PM pieces.

In order to improve the torque performance, an improved MVG with independent magnetizing windings is developed [15]. Fig. 1(b) shows the basic configuration of the improved MVG which consists of the inner rotor with surface mounted PM pieces, the stationary ring, the double-deck independent magnetizing windings and the outer rotor surface mounted with PM pieces. Its key design data is also listed in Table I. By carefully selecting the number of iron segments in the stationary ring, namely 19, the improved MVG can be designed to offer six sets of gear ratios, namely from 0.27 to 3.75 . In order to reduce the negative influence caused by the introduction of magnetizing windings, the double-deck magnetizing windings are located in the air part of the stationary ring. As shown in Fig. 1(b), the double-deck magnetizing windings are surrounded by iron components while air bridges are inserted between them. The air bridge functions to decrease the flux leakage during normal operation, hence reducing the torque ripple. However, the structure becomes very complicated and the magnetizing current is still high.

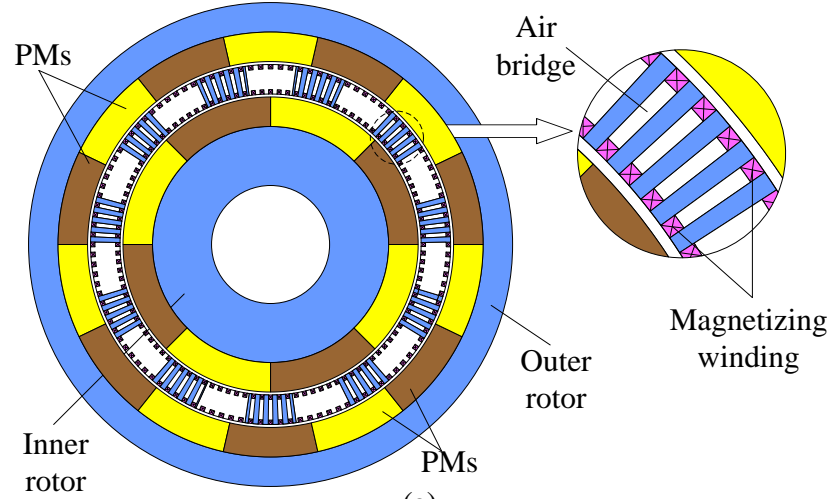

(a)

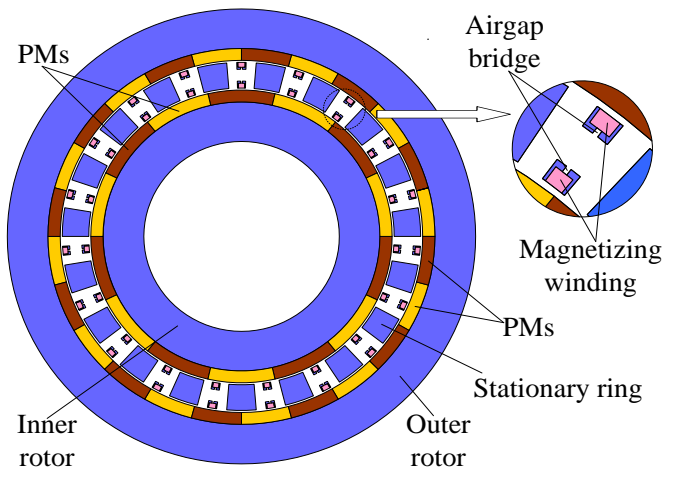

(b)

Fig. 1. Configuration of MVGs: (a) original MVG; (b) improved MVG 
TABLE I. DESIGN PARAMETERS OF THREE MVGS

\begin{tabular}{lccc}
\hline \hline Items & Original MVG & Improved MVG & Proposed MVG \\
\hline Outer rotor outside diameter & $204 \mathrm{~mm}$ & $230 \mathrm{~mm}$ & $230 \mathrm{~mm}$ \\
Outer rotor inside diameter & $144 \mathrm{~mm}$ & $190 \mathrm{~mm}$ & $180 \mathrm{~mm}$ \\
Inner rotor outside diameter & $80 \mathrm{~mm}$ & $136 \mathrm{~mm}$ & $152 \mathrm{~mm}$ \\
Inner rotor inside diameter & $40 \mathrm{~mm}$ & $96 \mathrm{~mm}$ & $80 \mathrm{~mm}$ \\
No. of iron segments in stationary ring & 11 & 19 & 23 \\
No. of pole-pairs in outer rotor & $8 \sim 3$ & $4,5,7,12,14,15$ & $19 \sim 4$ \\
No. of pole-pairs in inner rotor & $3 \sim 8$ & $15,14,12,7,5,4$ & $4 \sim 19$ \\
No. of upper-deck and lower deck & 112 & 19 & 23 \\
magnetizing windings & $0.38 \sim 2.67$ & $0.27 \sim 3.75$ & $0.21 \sim 4.75$ \\
Gear ratio range & 6 & 6 & 16 \\
No. of gear ratios & $1.05 \mathrm{~T}$ & $1.05 \mathrm{~T}$ & $1.05 \mathrm{~T}$ \\
Remanence of Alnico PM & & & \\
\hline
\end{tabular}

\section{Proposed Magnetic VARiable Gear}

Although the original MVG and improved MVG can offer several gear ratios to adapt different driving requirements and road conditions, they suffer from many drawbacks as aforementioned. The key of the proposed MVG is to artfully arrange the magnetizing windings in the stationary ring so that it can allow for a wide range of numbers of the PM pole-pairs and hence a wide range of changeable gear ratios, while solving the drawbacks of high torque ripple and high magnetizing current. In this section, the topology of the proposed MVG is presented and then the theoretical analysis is conducted. In addition, the proposed MVG applied in hybrid propulsion system is discussed.

\section{A. Proposed MVG topology}

Fig. 2(a) depicts the $2 \mathrm{D}$ and $3 \mathrm{D}$ views of the topology of the proposed MVG which consists of the outer rotor surface mounted with PM pieces, the stationary ring integrated with the double-deck magnetizing windings and the inner rotor surface mounted with PM pieces. The key is to artfully accommodate the double-deck magnetization windings in the stationary ring in such a way the Alnico PM pieces can be dynamically magnetized or demagnetized simultaneously using the double-deck magnetizing windings, hence achieving the ability of gear-ratio-changing. The key design parameters of this MVG are also listed in Table I. 


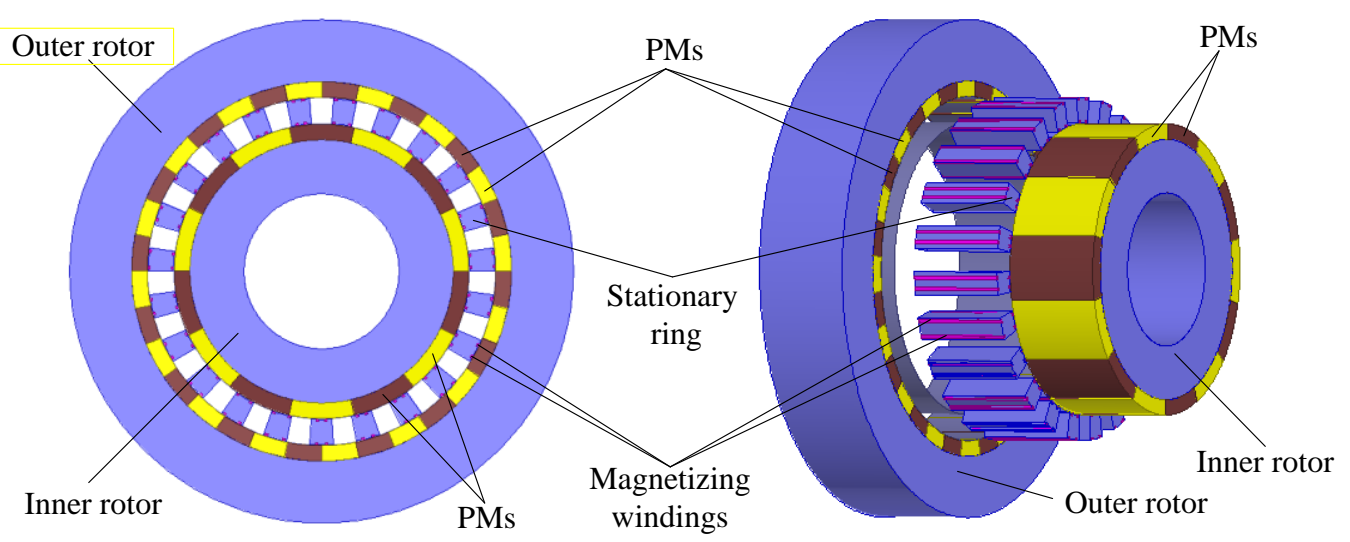

(a)

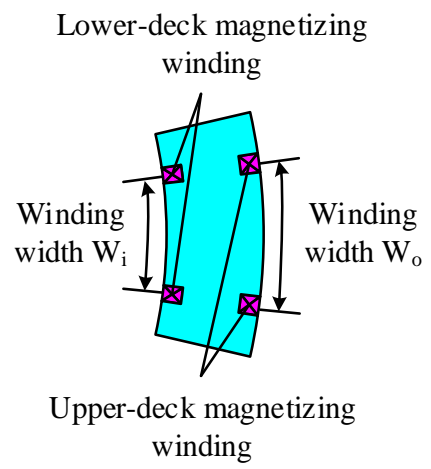

(b)

Fig. 2. Configuration of the proposed MVG: (a) overall structure; (b) stationary ring structure

Firstly, in order to achieve the function of online magnetization or demagnetization, the high-remanence low-coercivity Alnico is the key to play this role. Although the low-coercivity makes Alnico vulnerable to accidental demagnetization by armature field in conventional machines, this problem does not occur in the proposed MVG because it does not involve any armature current after the gear-ratio-changing process. Although the rare-earth PM based magnetic gears have better electromagnetic performances than the non-rare-earth based magnetic gears, the utilization of non-rare-earth Alnico for magnetic gears offers the advantage of better cost-effectiveness than the rare-earth PM materials such as the NdFeB and SmCo. Also, the Alnico PM offers the definite advantage of high Curie temperature which is essential when working at harsh vehicular environment.

Secondly, by purposely selecting the number of iron segments in the stationary ring, namely 23 , the proposed MVG can offer sixteen sets of gear ratios, namely from 0.21 to 4.75 . Considering the fact that the proposed MVG is directly connected to the wheel to drive the vehicle whereas the traditional mechanical box (generally offers seven gear ratios) is connected to the output shaft through speed reducer, hence the proposed MVG needs more gear ratios to accurately fulfill drive requirements. However, according to the design principle (equation 14), the more gear ratios of the MVG design, the higher the complexity is resulted. If the number of gear ratios is determined as 12 , the configuration of the MVG is simplified whereas the drive requirements can not 
be perfectly fulfilled. If the number of the gear ratios is selected as 20 , the problem of the drive requirements can be solved whereas complicated configuration is resulted. In order to balance the configuration complexity and drive requirements, the number of gear ratios is designed as 16 . Furthermore, in order to enhance the online magnetization capability, the double-deck magnetization windings are artfully inserted into the iron segments of the stationary ring as depicted in Fig. 2(b). During the gear-ratio-changing process, the lower-deck and upper-deck magnetizing windings are excited by the same magnetizing current to produce the same direction of magnetic field. Since both the lower-deck and upper-deck magnetizing windings are involved in the gear-ratio-changing process simultaneously, the required magnetizing current in the proposed MVG is at most one half of that in the improved MVG. Also, during normal operation, the magnetizing windings are open-circuited so that they will not incur magnetic field disturbing the torque transmission.

Thirdly, the key design parameters of the three MVGs, namely the number of magnetizing windings, the number of gear ratios and the gear ratio range, are quantitatively compared. As shown in Table I, the number of magnetizing windings in the proposed MVG is 23 , which is 4.9 times lower than that adopted in the original MVG, hence simplifying the structure of the stationary ring. Also, the proposed MVG can offer sixteen gear ratios, which is 2.7 times higher than that provided by the original MVG and the improved MVG. Moreover, the proposed MVG can offer the widest gear ratio range, namely 0.21 4.75. With these sixteen gear ratios, the proposed MVG can well fulfill different road conditions and driving requirements.

\section{B. Theoretical analysis}

The fundamental of the proposed MVG is the modulation of magnetic fields built up by the inner rotor PMs and the outer rotor PMs simultaneously. For simplicity, the magnetic saturation in iron teeth and yokes does not take into account. When the stationary ring is absent, the magnetic flux density distribution at a radial distance $r$ produced by the inner rotor and outer rotor are derived as two components [16]. The radial component is given by:

$$
\begin{aligned}
B_{r}(r, \Delta \theta) & =\underbrace{\frac{p_{o} b_{r m}}{p_{o}-1} \frac{1-R_{c}^{p_{o}-1} R_{d}{ }^{1-p_{o}}}{1-R_{a}{ }^{2 p_{o}} R_{d}{ }^{-2 p_{i}}}\left[R_{c}^{1-p_{o}} r^{p_{o}-1}+R_{a}^{2 p_{o}} R_{c}^{1-p_{o}} r^{-p_{o}-1}\right] \cos \left(-p_{o} \omega_{o} t+p_{o} \Delta \theta\right)}_{\text {flux densitydistribution producedby outerrotorPMs }} \\
+ & \underbrace{\frac{p_{i} b_{r m}}{1+p_{i}} \frac{1-R_{a}^{p_{i}+1} R_{b}{ }^{-p_{i}-1}}{1-R_{a}{ }^{2 p_{i}} R_{d}^{-2 p_{i}}}\left[R_{b}^{p_{i}+1} R_{d}{ }^{-2 p_{i}} r^{p_{i}-1}+R_{b}^{p_{i}+1} r^{-p_{i}-1}\right] \cos \left(-p_{i} \omega_{i} t+p_{i} \Delta \theta\right)}_{\text {flux densitydistribution producedby innerrotor PMs }}
\end{aligned}
$$

The circumferential component is given by:

$$
B_{\theta}(r, \Delta \theta)=\underbrace{\frac{p_{o} b_{r m}}{1-p_{o}} \frac{1-R_{c}^{p_{o}-1} R_{d}^{1-p_{o}}}{1-R_{a}^{2 p_{o}} R_{d}{ }^{2 p_{i}}}\left[R_{c}^{1-p_{o}} r^{p_{o}-1}+R_{a}^{2 p_{o}} R_{c}^{1-p_{o}} r^{-p_{o}-1}\right] \sin \left(-p_{o} \omega_{o} t+p_{o} \Delta \theta\right)}_{\text {flux densitydistribution producedby outerrotor PMs }}
$$




$$
+\underbrace{\frac{p_{i} b_{r m}}{-p_{i}-1} \frac{1-R_{a}^{p_{i}+1} R_{b}^{-p_{i}-1}}{1-R_{a}^{2 p_{i}} R_{d}^{-2 p_{i}}}\left[R_{b}^{p_{i}+1} R_{d}^{-2 p_{i}} r^{p_{i}-1}+R_{b}^{p_{i}+1} r^{-p_{i}-1}\right] \sin \left(-p_{i} \omega_{i} t+p_{i} \Delta \theta\right)}_{\text {flux densitydistribution producedby innerrotor PMs }}
$$

where $R_{a}, R_{b}$ are the inside and outside radii of the inner rotor PM respectively; $R_{c}, R_{d}$ are the inside and outside radii of the outer rotor PM respectively; $p_{i}, p_{o}$ are the pole-pair numbers of the inner rotor and outer rotor PMs respectively; $\omega_{i}, \omega_{o}$ are the mechanical rotational speeds of the inner rotor and outer rotor respectively; and $\Delta \theta$ is the movement in mechanical angle.

When the stationary ring with double-deck magnetization windings is taken into consideration, the flux density in the iron segment is given by:

$$
B_{s}=B_{a} \lambda_{r}
$$

where $B_{a}$ is the flux density without the stationary ring; and $\ddot{e}_{r}$ is the relative airgap permeance. The flux density in the windings is the same as that in the air space. For the inner airgap, the radial component of $\lambda_{r i}$ can be expressed as:

$$
\lambda_{r i 1}=\sum_{n=0}^{\infty} \lambda_{n_{-} i a 1} \cos \left(n N_{s} \theta\right)
$$

Also, the circumferential component of $\ddot{e}_{r i}$ can be illustrated as:

$$
\lambda_{r i 2}=\sum_{m=0}^{\infty} \lambda_{m_{-} i a 2} \sin \left(m N_{s} \theta\right)
$$

Similarly, for the outer airgap, the radial and circumferential components of $\lambda_{r o}$ can be obtained as, respectively:

$$
\begin{aligned}
& \lambda_{r o 1}=\sum_{n=0}^{\infty} \lambda_{n_{-} o a 1} \cos \left(n N_{s} \theta\right) \\
& \lambda_{r o 2}=\sum_{m=0}^{\infty} \lambda_{m_{-} o a 2} \sin \left(m N_{s} \theta\right)
\end{aligned}
$$

Hence, the resultant flux density in the outer airgap inspired by the inner and outer rotors can be deduced. The corresponding radial component is given by:

$$
B_{r_{-} o a}\left(r_{o}, \Delta \theta\right)=\sum_{n=0}^{\infty} \lambda_{n_{-} o a 1} \cos \left(n N_{s} \theta\right) B_{r}\left(r_{o}, \Delta \theta\right)+\sum_{m=0}^{\infty} \lambda_{m_{-} o a 2} \sin \left(m N_{s} \theta\right) B_{\theta}\left(r_{o}, \Delta \theta\right)
$$

The corresponding circumferential component is given by:

$$
B_{\theta_{-} o a}\left(r_{o}, \Delta \theta\right)=\sum_{n=0}^{\infty} \lambda_{n_{-} o a 1} \cos \left(n N_{s} \theta\right) B_{\theta}\left(r_{o}, \Delta \theta\right)-\sum_{m=0}^{\infty} \lambda_{m_{-} o a 2} \sin \left(m N_{s} \theta\right) B_{r}\left(r_{o}, \Delta \theta\right)
$$

Similarly, the resultant flux density in the inner airgap inspired by the inner and outer rotors can be deduced. The corresponding radial component is given by: 


$$
B_{r_{-} i a}\left(r_{i}, \Delta \theta\right)=\sum_{n=0}^{\infty} \lambda_{n_{-} i a 1} \cos \left(n N_{s} \theta\right) B_{r}\left(r_{i}, \Delta \theta\right)+\sum_{m=0}^{\infty} \lambda_{m_{-} i a 2} \sin \left(m N_{s} \theta\right) B_{\theta}\left(r_{i}, \Delta \theta\right)
$$

The corresponding circumferential component is given by:

$$
B_{\theta_{-} i a}\left(r_{i}, \Delta \theta\right)=\sum_{n=0}^{\infty} \lambda_{n_{-} i a 1} \cos \left(n N_{s} \theta\right) B_{\theta}\left(r_{i}, \Delta \theta\right)-\sum_{m=0}^{\infty} \lambda_{m_{-} i a 2} \sin \left(m N_{s} \theta\right) B_{r}\left(r_{i}, \Delta \theta\right)
$$

where $N_{s}$ is the number of iron segments in the stationary ring. Consequently, the magnetic torque transmitted at the outer and inner airgaps can be respectively expressed as:

$$
\left\{\begin{array}{l}
T_{o}=\int_{0}^{2 \pi} \frac{h r_{o}^{2}}{\mu_{0}} B_{r_{-} o a} B_{\theta_{-} o a} d \theta \\
T_{i}=\int_{0}^{2 \pi} \frac{h r_{i}^{2}}{\mu_{0}} B_{r_{-} i a} B_{\theta_{-} i a} d \theta
\end{array}\right.
$$

where $h$ is the axial length of the proposed MVG; and $\mu_{0}$ is the permeability of vacuum.

In order to obtain the ability of gear-ratio-changing, the newly designed stationary ring should possess the function of variable gear ratios. Based on the derivation for conventional coaxial magnetic gears [7], the operation principle is governed by:

$$
\left\{\begin{array}{l}
N_{s}=p_{i}+p_{o} \\
G_{r}=-\frac{\omega_{i}}{\omega_{o}}=-\frac{p_{o}}{p_{i}}
\end{array}\right.
$$

where $G_{r}$ is the defined gear ratio; $\omega_{i}$ and $\omega_{o}$ are the rotational speeds of the inner and outer rotors respectively; and the minus notation means that the rotors run at opposite directions. The vital principle of the proposed MVG is the mathematical relationship among the pole-pair number of outer rotor PMs $p_{o}$, the pole-pair number of inner rotor PMs $p_{i}$, the number of inner rotor PM pieces $k_{i}$, the number of outer rotor PM pieces $k_{o}$, the number of upper-deck magnetizing windings $d_{o}$, the number of lower-deck magnetizing windings $d_{i}$ and the number of iron segments in the stationary ring $N_{s}$. Firstly, the number of the stationary ring $N_{s}$ should be a prime number because it can enable good magnetic flux modulation effect, hence resulting in low torque ripple. Secondly, when the $N_{s}$ is selected as constant, in order to adjust the gear ratio, the numbers of inner rotor and outer PM pieces are governed by: 


$$
\left\{\begin{array}{l}
X=(\underbrace{\left(\mathrm{G}_{1\left(\mathrm{~N}_{\mathrm{s}}-1\right)}, \mathrm{G}_{2\left(\mathrm{~N}_{\mathrm{s}}-2\right)}, \ldots \mathrm{G}_{\left(\mathrm{N}_{\mathrm{s}}-1\right) 1}\right.}_{\text {possiblegear ratio }}) \\
Y_{k\left(N_{s}-k\right)}=\left[N_{s}-k, k\right] \\
k_{i}=k_{o}=2 m[\underbrace{Y_{k_{1}\left(N_{s}-k_{1}\right)}, \ldots, Y_{\left(N_{s}-k_{1}\right) k_{1}}}_{\text {adoptedgear ratio }}] \\
1 \leq k=k_{1} \leq\left(N_{s}-1\right) \\
m=1,2,3, \ldots
\end{array}\right.
$$

where the array $X$ represents the possible gear ratios based on the selected $N_{s} ; Y_{k(N s-k)}$ is the least common multiple of $\left(N_{s}-k\right)$ and $k$; and $Y_{k_{1}\left(N_{s}-k_{1}\right)}$ is the necessary least number of PM pieces under the selected gear ratio. Then, the values of $d_{o}$ and $d_{i}$ are governed by:

$$
d_{o}=d_{i}=N_{s}
$$

In order to magnetize or demagnetize the PM pieces efficiently, the width of double-deck magnetizing windings is purposely designed. The width of the upper-deck magnetizing winding $W_{o}$ and the width of the lower-deck magnetizing winding $W_{i}$ are governed by:

$$
\left\{\begin{array}{l}
W o=\frac{\pi R_{o s}}{n p_{o}}<\frac{\pi R_{o s}}{N_{s}} \\
W i=\frac{\pi R_{i s}}{n p_{i}}<\frac{\pi R_{i s}}{N_{s}} \\
m=1,2,3, \ldots \\
n=1,2,3, \ldots
\end{array}\right.
$$

where $R_{o s}$ and $R_{i s}$ are the inside and outside radii of the stationary ring. Considering the practicability of gear-ratio-changing, $W_{o}$ and $W_{i}$ should be as large as possible.

\section{MVG based hybrid vehicular propulsion system}

Fig. 3 shows the configuration of the proposed MVG based hybrid propulsion system which consists of an engine, two electric machines, three converters and the proposed MVG. As shown in Fig. 3, the outer rotor of the proposed MVG is coupled with the engine via the machine 1 and the inner rotor is coupled with the driveline via the machine 2 . The machine 1 plays the role to perform the function of power-split of the engine. In other words, the machine 1 splits a portion of engine output in the form of mechanical power to the MVG and another portion in the form of electrical power to the converter 1 . Specifically, when the vehicle needs high power, the engine output will be directly transmitted to the driveline via the MVG and the machine 1 does not extract any power from the engine. If necessary, the battery feeds the machine 2 via the converter 2 to provide additional power to the driveline. On the other hand, when the vehicle needs only low power, the machine 1 will divert a large portion of 
the engine output and store it in the battery. The converter 3 serves to properly feed the magnetizing windings of the MVG. Hence the engine can always work at the optimal operating line no matter how much power is required at the driveline. It should be noted that the continuously variable transmission between the driveline and the engine is achieved by the power-split process, whereas the MVG functions to extend the torque and speed ranges.

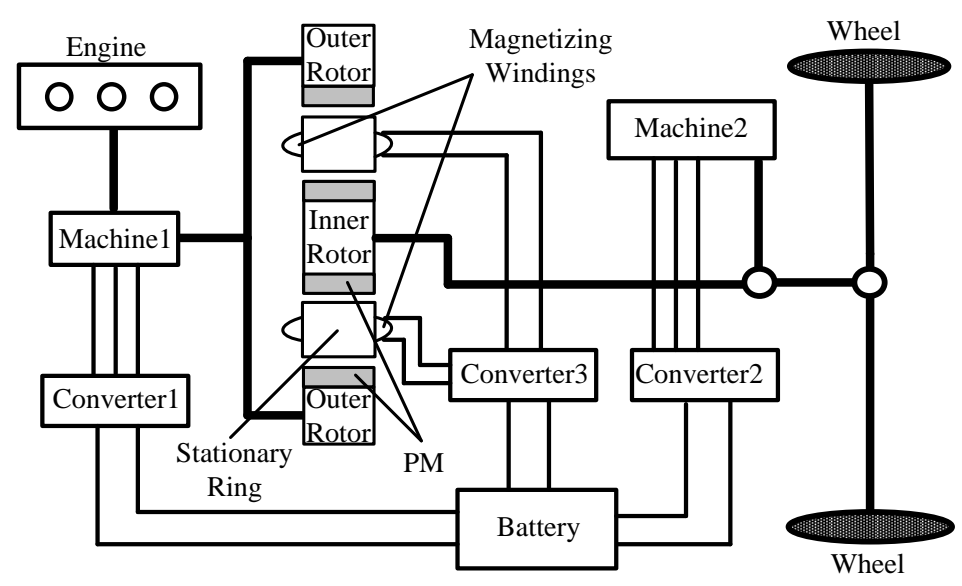

Fig. 3. MVG based hybrid vehicular propulsion system

By properly controlling the three converters, the proposed MVG based hybrid propulsion system can achieve various operating modes: 1) hybrid electric mode; 2) full electric mode; 3) regenerative braking mode. Fig.4 Fig.6 show the power flow of three operating modes in detail. As shown in Fig. 4, during the hybrid electric mode, both the power-split process of the machine 1 and the gear-ratio-changing process of the MVG are activated to provide wide-range continuously variable transmission, hence optimizing the engine efficiency over wide torque and speed ranges of the vehicle. As depicted in Fig.5, during the full electric mode, the engine does not work and the required vehicle power can be provided solely by the machine 2 which is fed by the battery via the converter 2, hence resulting in zero emission. As exhibited in Fig. 6, when the vehicle brakes or runs downhill, the machine 2 operates as a generator to capture the regenerative braking energy and charge the battery via the converter 2. In addition, the two machines take the charge of adjusting the two rotor positions of the MVG during the gear-ratiochanging process.

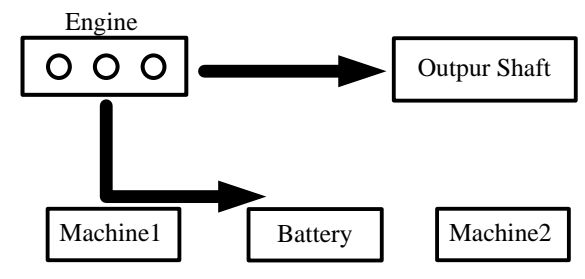

(a)

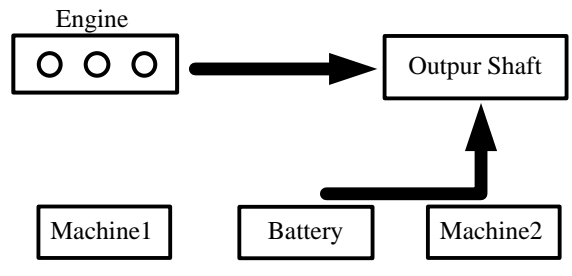

(b)

Fig. 4. Power flow chart of hybrid electric mode: (a) light load condition; (b) heavy load condition 


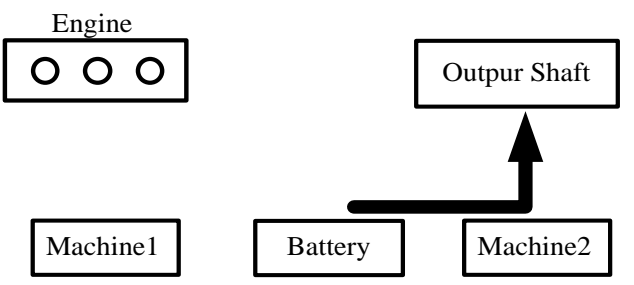

Fig. 5. Power flow chart of full electric mode

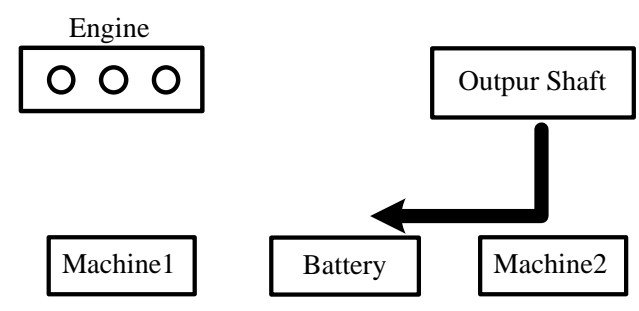

Fig. 6. Power flow chart of regenerative brake mode

Recently, magnetic gears have been further extended to electronic-continuously variable transmission (E-CVT) systems [17][18]. The key of these magnetic-geared E-CVT systems is the use of magnetic gear to perform power-split of the engine. Specifically, two machines are incorporated into the magnetic gear. One machine is responsible for the generation while the other machine is designed as a motor. Under light load condition, the power from the engine can be divided into two paths, namely driving the vehicle through the motor and storing extra power into the battery through the generator. Under heavy load condition, the motor and the engine are involved in the propulsion simultaneously. Hence, the relative speeds between the outer rotor, inner rotor and modulation ring of the magnetic gear are properly regulated to enable the engine working at the optimal operating line. In the proposed MVG based hybrid propulsion system, the task of power-split is separately handled by the machine 1 while the MVG serves to directly manipulate the mechanical power flow to the driveline. On one hand, the machine 1 extracts some power from the engine when the vehicle works at light load condition. On the other hand, under heavy load condition the machine 2 and engine are responsible for the propulsion at the same time. Thus, the output torque and speed ranges at the driveline can be further extended.

\section{RESUlts AND ANALYSiS}

In order to validate the theoretical analysis, the electromagnetic performances of the proposed MVG are analyzed. By online changing the numbers of PM pole-pairs in the inner and outer rotors, the gear ratio becomes controllable. According to (11), the parameters $d_{i}, d_{o}$ are selected as 23 and $N_{s}$ is selected as 23 so that $p_{i}$ spans from 4 to 19 while $p_{o}$ spans from 19 to 4 accordingly. Hence, $G_{r}=4 / 19, G_{r}=5 / 18, G_{r}=6 / 17, G_{r}=7 / 16, G_{r}=8 / 15, G_{r}=9 / 14, G_{r}=10 / 13, G_{r}=11 / 12, G_{r}=12 / 11, G_{r}=13 / 10, G_{r}=14 / 9, G_{r}=15 / 8$, $G_{r}=16 / 7, G_{r}=17 / 6, G_{r}=18 / 5$ and $G_{r}=19 / 4$ are the resulting sixteen sets of gear ratios. 
Firstly, the airgap flux density and steady-state torque of the proposed MVG under sixteen sets of $G_{r}$ are simulated. The inner and outer airgap flux density waveforms at $G_{r}=4 / 19$ are shown in Fig. 7(a) and Fig. 7(b), respectively. From the waveforms, it confirms that the number of inner airgap pole-pairs is 19 and the number of outer airgap pole-pairs is 4 , which are in accordance with the gear ratio of 4/19. Similarly, the corresponding waveforms under $G_{r}=6 / 17, G_{r}=8 / 15, G_{r}=10 / 13, G_{r}=12 / 11, G_{r}=14 / 9$, $G_{r}=16 / 7$, and $G_{r}=18 / 5$ are shown in Fig. 7 to Fig. 10, respectively. It can be found that the mathematical relationship between the pole-pair numbers at the inner rotor and outer rotor under each gear ratio well agrees with the value of the corresponding gear ratio. In addition, all steady-state torques and torque ripples under sixteen sets of $G_{\mathrm{r}}$ are tabulated in Table II. From the torque performance, it can be observed that the steady-state torques at the inner rotor and outer rotor under $G_{r}=4 / 19$ are $131.25 \mathrm{Nm}$ and $27.6 \mathrm{Nm}$, respectively, which agrees with the ratio of $4 / 19$. The corresponding torque ripples are $2.28 \%$ and $15.2 \%$, respectively. Similarly, the relationships between the steady-state torques at the inner rotor and outer rotor well agree with the gear ratios. Moreover, it can be observed that the maximum inner rotor torque ripple is only $6.58 \%$ which is very acceptable for driving the output shaft of vehicles. Although the maximum outer rotor torque ripple is relatively larger, up to $15.2 \%$, it does not cause significant adverse effects because the outer rotor is coupled with the engine and such vibration can readily be absorbed.

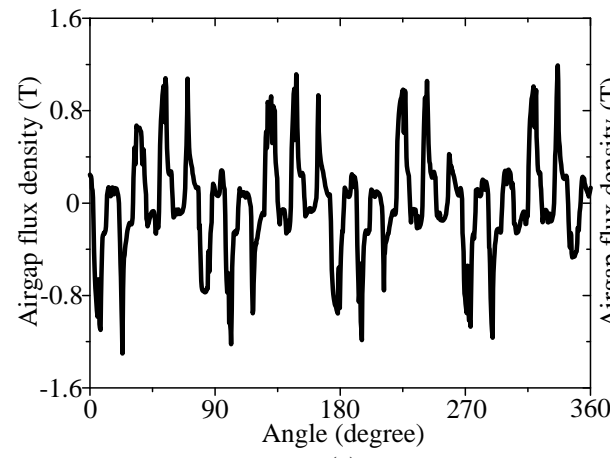

(a)

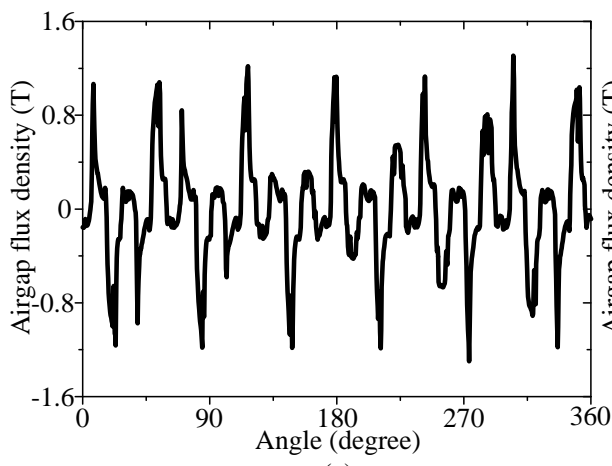

(a)

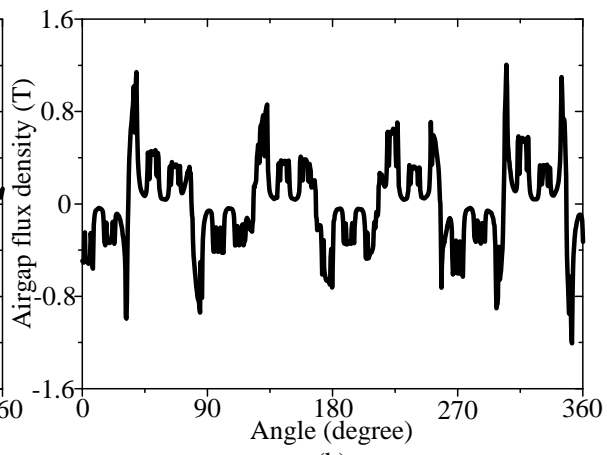

(b)

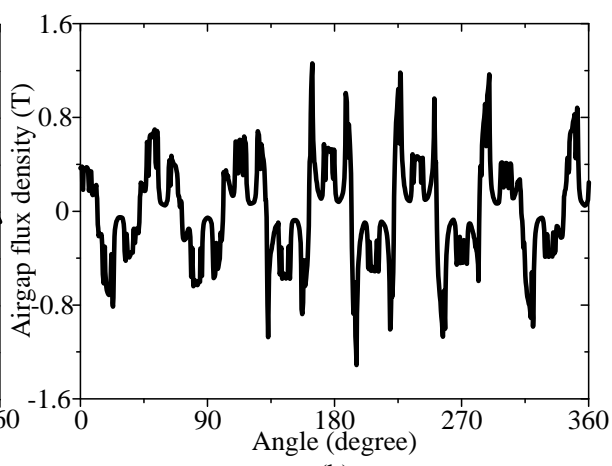

(b)

Fig. 7. Magnetic flux performances of MVG:

(a) Inner airgap flux density at $G_{r}=4 / 19$; (b) Outer airgap flux density at $G_{r}=4 / 19$;

(c) Inner airgap flux density at $G_{r}=6 / 17$; (d) Outer airgap flux density at $G_{r}=6 / 17$ 

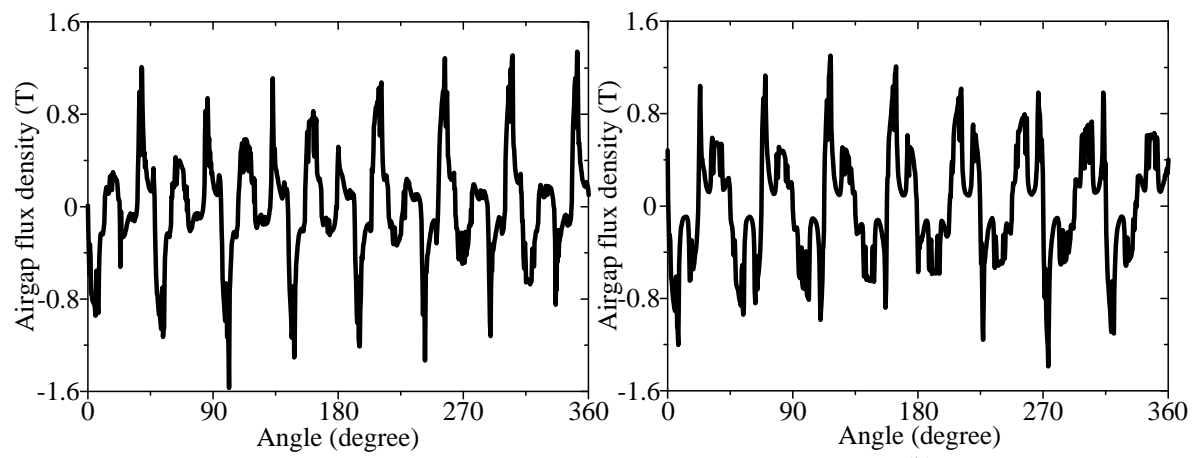

(a)

(b)

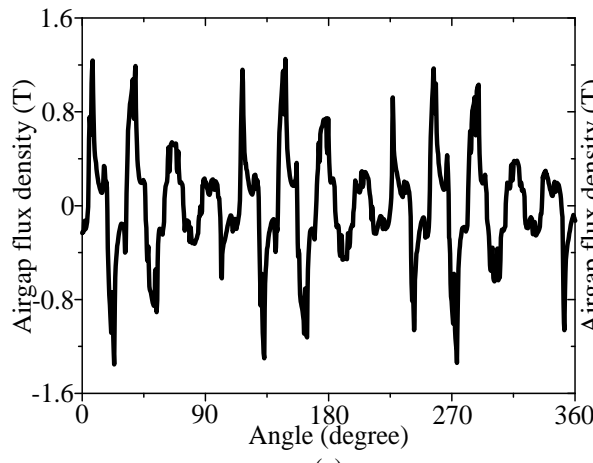

(a)

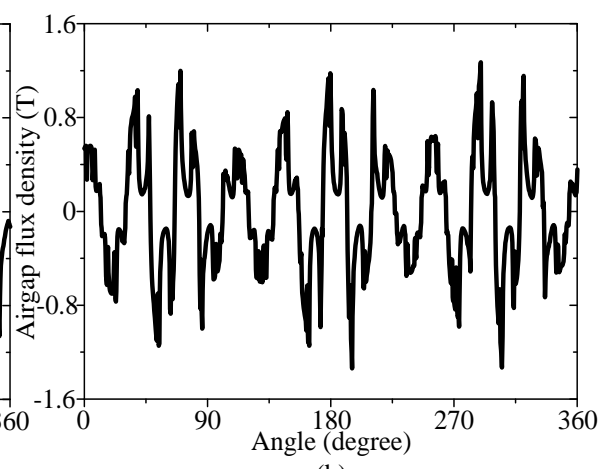

(b)

Fig. 8. Magnetic flux performances of MVG:

(a) Inner airgap flux density at $G_{r}=8 / 15$; (b) Outer airgap flux density at $G_{r}=8 / 15$;

(c) Inner airgap flux density at $G_{r}=10 / 13$; (d) Outer airgap flux density at $G_{r}=10 / 13$

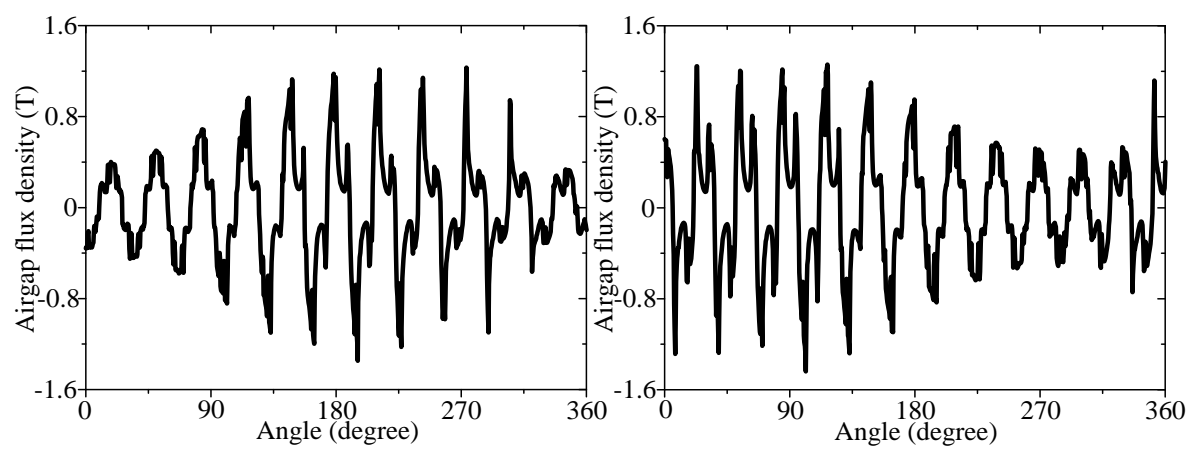

(a)

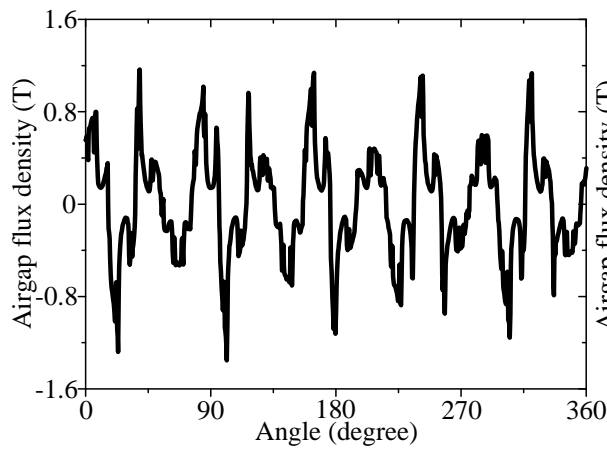

(b)

(a)

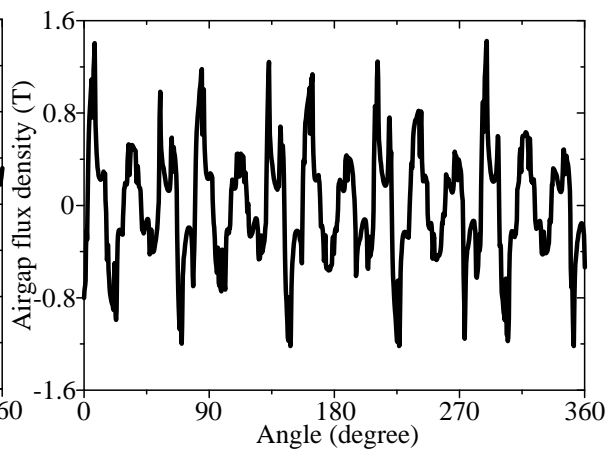

(b)

Fig. 9. Magnetic flux performances of MVG:

(a) Inner airgap flux density at $G_{r}=12 / 11$; (b) Outer airgap flux density at $G_{r}=12 / 11$;

(c) Inner airgap flux density at $G_{r}=14 / 9$; (d) Outer airgap flux density at $G_{r}=14 / 9$. 


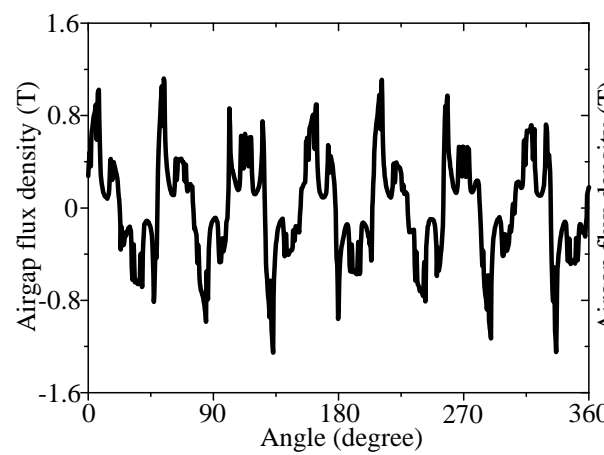

(a)

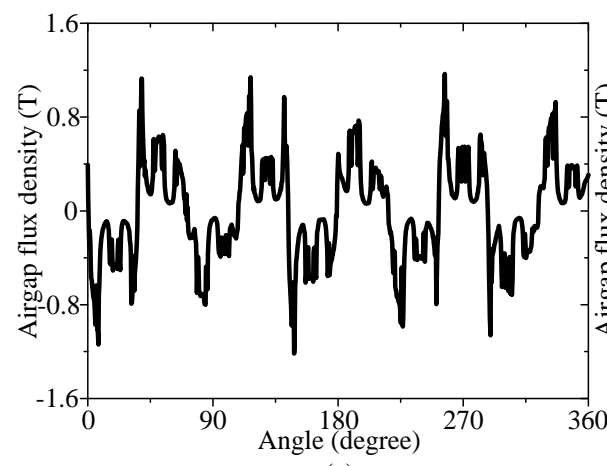

(a)

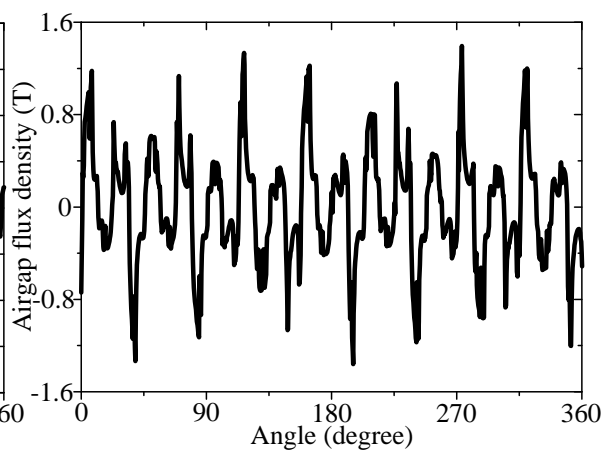

(b)

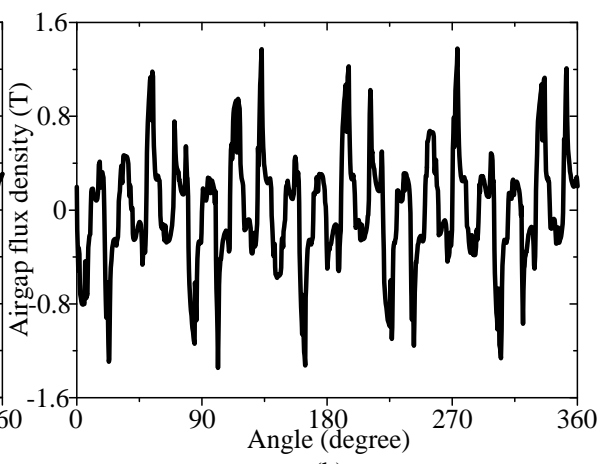

(b)

Fig. 10. Magnetic flux performances of MVG:

(a) Inner airgap flux density at $G_{r}=16 / 7$; (b) Outer airgap flux density at $G_{r}=16 / 7$;

(c) Inner airgap flux density at $G_{r}=18 / 5$; (d) Outer airgap flux density at $G_{r}=18 / 5$

TABLE II. TORQUE PERFORMANCE OF PROPOSED MVG

\begin{tabular}{ccccc}
\hline \hline $\begin{array}{c}\text { Gear } \\
\text { ratio }\end{array}$ & $\begin{array}{c}\text { Outer rotor } \\
\text { steady-state } \\
\text { torque [Nm] }\end{array}$ & $\begin{array}{c}\text { Outer rotor } \\
\text { torque } \\
\text { ripple }\end{array}$ & $\begin{array}{c}\text { Inner rotor } \\
\text { steady-state } \\
\text { torque [Nm] }\end{array}$ & $\begin{array}{c}\text { Inner rotor } \\
\text { torque } \\
\text { ripple }\end{array}$ \\
\hline $4 / 19$ & 27.6 & $15.2 \%$ & 131.25 & $2.28 \%$ \\
$5 / 18$ & 40.5 & $12.3 \%$ & 143.5 & $2.78 \%$ \\
$6 / 17$ & 54.5 & $9.17 \%$ & 154.5 & $2.59 \%$ \\
$7 / 16$ & 70.5 & $7.09 \%$ & 161 & $2.48 \%$ \\
$8 / 15$ & 87.4 & $1.89 \%$ & 164.2 & $1.7 \%$ \\
$9 / 14$ & 105.5 & $4.97 \%$ & 164 & $2.13 \%$ \\
$10 / 13$ & 122.5 & $5.71 \%$ & 159.5 & $2.19 \%$ \\
$11 / 12$ & 140 & $3.57 \%$ & 152.7 & $2.6 \%$ \\
$12 / 11$ & 156.2 & $4.48 \%$ & 143 & $3.15 \%$ \\
$13 / 10$ & 171 & $2.92 \%$ & 131.2 & $2.9 \%$ \\
$14 / 9$ & 182.5 & $3.84 \%$ & 117.5 & $2.98 \%$ \\
$15 / 8$ & 192 & $1.04 \%$ & 102 & $1.47 \%$ \\
$16 / 7$ & 196 & $3.06 \%$ & 86 & $3.48 \%$ \\
$17 / 6$ & 195.8 & $4.59 \%$ & 69 & $4.35 \%$ \\
$18 / 5$ & 190.8 & $3.25 \%$ & 53 & $6.28 \%$ \\
$19 / 4$ & 180 & $2.89 \%$ & 38 & $6.58 \%$ \\
\hline
\end{tabular}

Secondly, the torque transmission capability of the proposed MVG under all gear ratios are simulated, hence obtaining the corresponding torque-angle curves. Table III summaries the torque transmission capability of the proposed MVG under all gear ratios. As shown in Table III, it confirms that the maximum torque developed at the outer rotor is $28.6 \mathrm{Nm}$ and the maximum torque developed at the inner rotor is $135.5 \mathrm{Nm}$ under the $G_{r}=4 / 19$, which well agrees with the gear ratio of $4 / 19$. Also, it is noted 
that the maximum torque transmitted by the outer rotor is $40.8 \mathrm{Nm}$ under the $G_{r}=5 / 18$, which is 3.6 times lower than the maximum torque transmitted by the inner rotor, validating the corresponding gear ratio of 5/18. Consequently, it can be observed that the relationships between the maximum torques at the inner rotor and outer rotor under all sixteen gear ratios well agree with the corresponding gear ratios, respectively. Furthermore, under the torque-amplification gear ratios, namely from $G_{r}=4 / 19$ to $G_{r}=11 / 12$, the output torques are amplified steadily so that these gear ratios are more preferable for heavy-load conditions such as vehicle launching or hill-climbing. Meanwhile, under the speed-amplification gear ratios, namely from $G_{r}=12 / 11$ to $G_{r}=19 / 4$, the output speeds are amplified steadily so that these gear ratios are more preferable for high-speed operations such as vehicle cruising. Moreover, the torque density of this MVG is $24.76 \mathrm{kN} / \mathrm{m}^{3}$ which is very acceptable for vehicular propulsion transmission. With these sixteen gear ratios, namely from 0.21 to 4.75 , the vehicle can provide almost continuously variable gear ratios to extend the torque and speed ranges for different driving requirements and road conditions.

TABLE III. TORQUE TRANSMISSION CAPABILITY OF PROPOSED MVG

\begin{tabular}{ccc}
\hline \hline Gear ratio & $\begin{array}{c}\text { Outer rotor maximum } \\
\text { torque }[\mathbf{N m}]\end{array}$ & $\begin{array}{c}\text { Inner rotor maximum torque } \\
{[\mathbf{N m}]}\end{array}$ \\
\hline $4 / 19$ & 28.6 & 135.5 \\
$5 / 18$ & 40.8 & 145.6 \\
$6 / 17$ & 56.2 & 159.7 \\
$7 / 16$ & 71.5 & 163.7 \\
$8 / 15$ & 90.2 & 168.7 \\
$9 / 14$ & 108.8 & 169.5 \\
$10 / 13$ & 123.2 & 160.5 \\
$11 / 12$ & 144.8 & 157.8 \\
$12 / 11$ & 156.3 & 143.1 \\
$13 / 10$ & 172.5 & 132.5 \\
$14 / 9$ & 188.2 & 120.9 \\
$15 / 8$ & 191.8 & 102.5 \\
$16 / 7$ & 202.5 & 88.1 \\
$17 / 6$ & 205.5 & 72.3 \\
$18 / 5$ & 196.2 & 54.8 \\
$19 / 4$ & 183.3 & 38.9 \\
\hline
\end{tabular}

Thirdly, a comparative study of the proposed MVG with and without the magnetizing windings is carried out. It can be found that the torque developed at the outer rotor is $205.6 \mathrm{Nm}$ under the $G_{r}=16 / 7$ if the magnetizing windings are absent. And the corresponding torque will reduce to $196 \mathrm{Nm}$ when taking into account the magnetizing windings, which is in accordance with the theoretical analysis. Although the transmission torque inevitably decreases with the introduction of magnetizing windings, the proposed MVG can still offer the torque density of $24.76 \mathrm{kN} / \mathrm{m}^{3}$. Meanwhile, the corresponding torque ripple produced at the outer rotor will increase from $0.48 \%$ to $3.06 \%$ with the introduction of magnetizing windings, hence further validating the theoretical analysis. It should be noted that this small vibration can readily be adsorbed by the engine shaft.

Fourthly, in order to illustrate the process of gear-ratio-changing, the changing of gear ratios from $G_{r}=4 / 3$ to $G_{r}=3 / 4$ is adopted for exemplification. According to the theoretical analysis, the parameters $d_{o}, d_{i}, k_{i}$ and $k_{o}$ are selected as 7, 7, 24 and 24 
respectively. The number of turns of the upper-deck and lower-deck magnetizing windings is set to 10 . The gear-ratio-changing process commences when the existing gear ratio differs from the targeted gear ratio. Then the corresponding PM pieces that need polarity changes are calculated accordingly. And the two machines adjust the positions of the PM pieces to face the relevant magnetizing windings. Hence, the converter 3 properly feeds the magnetizing current to effectuate the magnetization or demagnetization. Fig. 11 shows the pole-changing process of one PM piece. It can be observed that the magnetic polarity of PM pieces $\mathrm{I} 1$ and $\mathrm{O} 1$ are changed together when the magnetizing current per turn increases from $0 \mathrm{~A}$ to $1.5 \mathrm{~A}$. Also, it exhibits that the magnetic polarities of other PM pieces adjacent to the PM pieces I1 and O1 do not change, hence achieving independent pole-changing control. Moreover, the required magnetizing current is only a temporary current pulse which takes only 1-4 A for 50-100 ms. After all required PM pieces finishing the pole-changing, the MVG exhibits the targeted gear ratio.

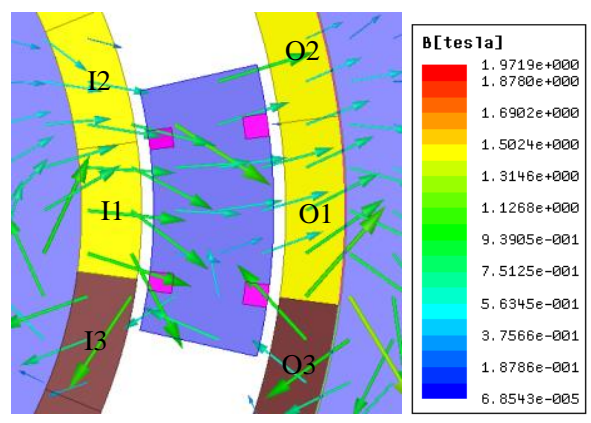

(a)

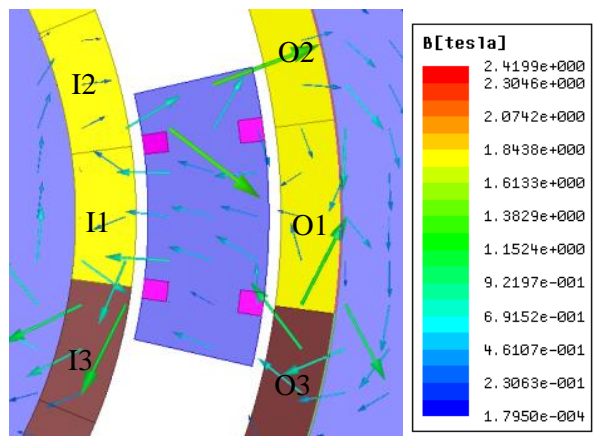

(c)

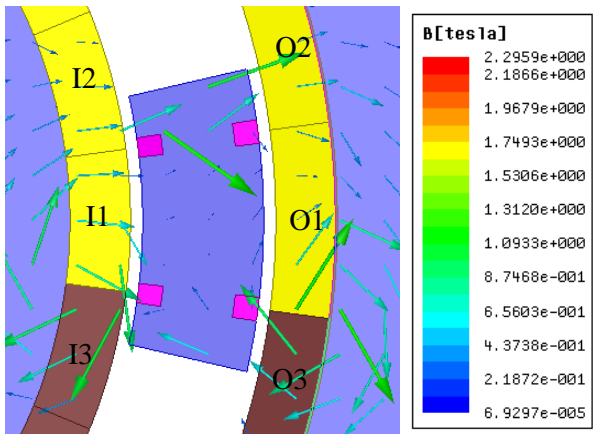

(b)

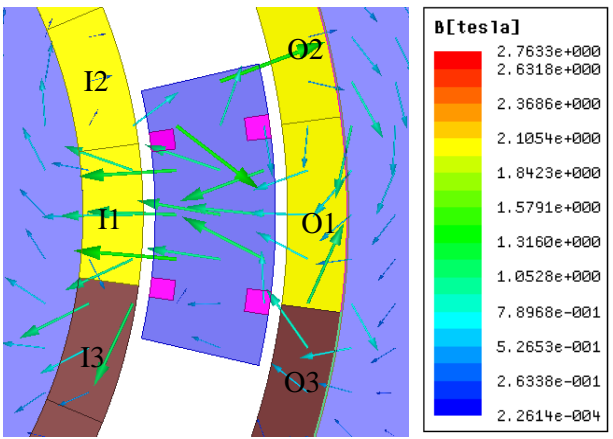

(d)

Fig. 11. Pole-changing process of one PM piece:

(a) Current per turn $=0 \mathrm{~A}$; (b) Current per turn=0.5A;

(c) Current per turn=1.0A; (d) Current per turn=1.5A

Finally, a quantitative comparison among the original MVG, the improved MVG and the proposed MVG are conducted. Table VI summarizes their numbers of gear ratios, torque densities, average output torque ripples and required magnetizing currents. As shown in Table IV, the proposed MVG offers the much more gear ratios, namely 16, whereas the original MVG and the improved MVG can only have six gear ratios. It indicates that the proposed MVG can offer much smoother change of torque and speed ranges. In addition, the proposed MVG possesses the highest torque density which is $205 \%$ of the improved MVG and 809\% of the original MVG. It means that the proposed MVG is the most suitable one for vehicular application. The original 
MVG exhibits the largest torque ripple, namely $21.3 \%$, which will cause undesirable negative effects on the output shaft. Although the average output torque ripple of the proposed MVG is slightly larger than that of the improved MVG, the required magnetizing current is much lower which it more practicable to achieve the desired gear-ratio-changing. Moreover, such 2-3\% torque ripple can readily be absorbed by the output shaft. Therefore, the proposed MVG is the preferred one for the hybrid vehicular propulsion system.

TABLE IV. QUANTITATIVE COMPARISON AMONG THREE MVGS

\begin{tabular}{ccccc}
\hline \hline Items & No. of gear ratios & Torque density & $\begin{array}{c}\text { Average output } \\
\text { torque ripple }\end{array}$ & Magnetizing current \\
\hline $\begin{array}{c}\text { Original } \\
\text { MVG }\end{array}$ & 6 & $3.06 \mathrm{kN} / \mathrm{m}^{3}$ & $21.3 \%$ & $10 \mathrm{~A}$ \\
\hline Improved MVG & 6 & $12.04 \mathrm{kN} / \mathrm{m}^{3}$ & $2 \%$ & $6 \mathrm{~A}$ \\
\hline Proposed MVG & 16 & $24.76 \mathrm{kN} / \mathrm{m}^{3}$ & $3.05 \%$ & $1.5 \mathrm{~A}$ \\
\hline
\end{tabular}

\section{CONCLUSION}

This paper has presented the design of a new non-rare-earth MVG which combines the concept of the magnetic gear and the concept of the memory machine. The key is to artfully incorporate the magnetizing windings into the stationary ring in such a way that sixteen sets of gear ratios can be achieved. The simulation results confirm that the proposed MVG offer online changeable sixteen gear ratios. The proposed MVG can achieve the torque density of $24.76 \mathrm{kN} / \mathrm{m}^{3}$, and the maximum torque ripple at the outer rotor that is connected to the output shaft is only $6.58 \%$.

Undoubtedly, the proposed MVG offers the benefits of contact free, silent operation and no need of regular maintenance, hence eliminating the contact-transmission problems aroused by the mechanical gearbox. While the proposed MVG based ECVT system can offer the property of continuously variable transmission which is similar to other E-CVT systems, the proposed MVG provides the additional flexibility of changeable gear ratios which can significantly extend the torque and speed ranges of the whole system, hence better fulfilling different driving requirements and road conditions. However, the proposed MVG still has the limitations of relatively complicated structure and demanding manufacture requirement.

\section{ACKNOWLEDGMENT}

This work was supported in part by a grant (Project No. 710612E) from Hong Kong Research Grants Council, Hong Kong Special Administrative Region, China, and a grant (Project No. JCYJ20120831142942515) from Basic Research Program of Science, Technology and Innovation Commission of Shenzhen Municipality (SZSTI), China. 


\section{REFERENCES}

[1] Chau, K.T., Chan, C.C.: 'Emerging energy-efficient technologies for hybrid electric vehicles', Proc. IEEE, 2007, 95, (4), pp. $821-835$

[2] Ehsani, M., Gao, Y., Emadi, A.: 'Modern Electric, Hybrid Electric, and Fuel Cell Vehicles: Fundamentals, Theory, and Design', CRC Press, 2010

[3] Chan, C.C., Jian, L., Dan, Tu, D.: 'Smart charging of electric vehicles - integration of energy and information' IET Electr. Systems Transp., 2014, 4, (4), pp. 89-96

[4] Mi, C., Masrur, M.A., Gao, D.W.: 'Hybrid Electric Vehicles: Principles and Applications with Practical Perspectives', Wiley, 2011

[5] Miller, J.M.: 'Propulsion Systems for Hybrid Vehicles', IET, 2011

[6] Chau, K.T.: 'Electric Vehicle Machines and Drives: Design, Analysis and Application’, Wiley-IEEE Press, 2015

[7] Atallah, K., Calverley, S.D., Howe, D.: 'Design, analysis and realization of a high-performance magnetic gear', IEE Proc. - Electr. Power Appl., 2004, 151, (2), pp. 135-143

[8] Jian, L., Chau, K.T., Gong, Y., et al.: 'Comparison of coaxial magnetic gears with different topologies', IEEE Trans. Magn. 2009, 45, (10), pp. 4526-4529

[9] Huang, C.C., Tsai, M.C., Dorrell, D.G., et al.: 'Development of a magnetic planetary gearbox', IEEE Trans. Magn., 2012, 44, (3), pp. 403-412

[10] Chen, M., Chau, K.T., Li, W., et al.: 'Development of non-rare-earth magnetic gears for electric vehicles,' Jour. of Asia. Elec. Vehi., 2012, 10, (2), pp. 1607-1613

[11] Ostovic, V.: 'Memory motors', IEEE Ind. Appl. Mag., 2003, 9, (1), pp. 52-61

[12] Yu, C., Chau, K.T.: 'Design, analysis, and control of DC-excited memory motors', IEEE Trans. Energy Convers., 2011, 26, (2), pp. 479-489

[13] Yu, C., Chau, K.T.: 'New fault-tolerant flux-mnemonic doubly-salient permanent-magnet motor drive', IET Electr. Power Appl., 2011, 5, (5), pp. 393-403

[14] Chen, M., Chau, K.T., Li, W., et al.: 'Design and analysis of a new magnetic gear with multiple gear ratios', IEEE Trans. Appl. Supe., 2010, 24, (3), pp. 0501904:1-4

[15] Chen, M., Chau, K.T., Liu, C.: 'Design and analysis of an advanced magnetic gear for hybrid electric vehicles,' Proc. of the IEEE Int. Magn. Conf. 2015, Beijing, China, May 2015, no. AU-13

[16] Jian, L., Chau, K.T., Gong, Y., et al.: 'Analytical calculation of magnetic field in surface-inset permanent magnet motors', IEEE Trans. Magn., 2009, 45, (10), pp. 4688-4691 
[17] Wang, J., Atallah, K., Carvley, S.D.: 'A magnetic continuously variable transmission device', IEEE Trans. Magn., 2011, 47, (10), pp. 2815-2818

[18] Jian, L., Chau, K.T.: 'Design and analysis of a magnetic-geared electronic-continuously variable transmission system using finite element method,' Prog. Elec. Rese., 2010, 107, pp. 47-61 